$\mathrm{DTP} / 99 / 51$

KCL-MTH-99-31

\title{
Electric Black Holes in Type 0 String Theory
}

\author{
Ivo Sachs \\ Department of Mathematical Sciences \\ University of Durham \\ Science Site, Durham DH1 3LE, UK \\ and \\ Department of Mathematics, King's College London \\ The Strand, London WC2R 2LS, UK
}

\begin{abstract}
We discuss $A d S_{2+1}$ (BTZ) black holes arising in type 0 string theory corresponding to D1-D5 and F1-NS5 bound states. In particular we describe a new family of non-dilatonic solutions with only $D p_{+}$, that is "electric" branes. These solutions are distinguished by the absence of fermions in the world volume theory which is an interacting CFT. They can not be obtained as a projection of a type II BPS-configuration. As for previous type 0 backgrounds linear stability is guaranteed only if the curvature is of the order of the string scale where $\alpha^{\prime}$ corrections cannot be excluded. Some problems concerning the counting of states are discussed.
\end{abstract}




\section{Introduction}

String theory has lead to important new insights in the microscopic properties of those black holes for which the near horizon geometry consists of an Anti-de Sitter $(A d S)$ throat times a sphere which represents the horizon [1]. In the case of $A d S_{2+1}$ the entropy and Hawking radiation can be exactly reproduced within the world volume theory of the corresponding brane configuration of type II string theory which in this case is a 1+1-dimensional conformal field theory (CFT). In fact, many of the properties of this CFT, in particular, the central charge can be obtained solely by analysing the generators of the asymptotic symmetries of the near horizon geometry [2] (see [3] for implications for the black hole entropy). These properties are therefore universal and essentially independent of the underlying microscopic theory. In fact $A d S_{2+1}$ gravity itself without any extra fields provides the simplest representation of the conformal algebra on the boundary [2, 4], but there are indications that gravitational degrees of freedom alone cannot account for for all micro states needed to produce the geometric entropy of black holes [5]. Another representation of the algebra is, of course, provided by the superconformal world volume theory of the $D 1 D 5$-brane system of type II theory [1, 6]. So far this is essentially the only realisation for which it has been possible to count the micro states explicitly. In that sense string theory passes a non-trivial test for a consistent microscopic theory of gravity. One of the puzzles raised by this is the role of supersymmetry, or the necessity of fermions in any consistent theory of gravity. It is therefore of interest to analyse black holes in theories without space-time supersymmetry from this point of view. As mentioned above pure gravity provides one such theory but does not seem to be able to account for all degrees of freedom.

In this paper we consider type 0 string theory which is distinguished by being supersymmetric on the world sheet but has no space-time fermions in the perturbative closed-string sector. As we shall see under suitable assumptions about higher order corrections there are black hole solutions that are indistinguishable from their type II cousins. The world volume theory, however, is rather different due to the absence of fermions. The main focus of this paper is to find consistent solutions to the type 0 low energy gravitational effective action and to identify the corresponding world volume theory. Previous work on the $A d S_{p+1} / C F T$ correspondence in type 0 theories includes [7, 8]. In particular we use the results of [7] where the low energy effective action has been determined up to second order in the tachyon. In 8$]$ the $D 1_{ \pm} D 5_{ \pm}$black hole solution was given and the entropy was reproduced within the corresponding world volume theory which is, in fact, a $\mathbf{Z}_{2}$ projection of the type IIB theory. This solution was further analysed in [9]. In this paper we shall describe a new solution which consists of only $D p_{+}$(i.e. 'electric') branes. The fact that such a configuration leads to a non-dilatonic (that is conformal) background may come as a surprise. Indeed generically both electric- and magnetic RR-fields need to be turned on in order to cancel the tachyon tadpole. However, as we shall see, for the $D 1 D 5$-brane system this is not so. Another important feature that distinguishes the $D 1_{+} D 5_{+}$black hole constructed here is that it cannot be obtained by a projection of a corresponding type II solution. In particular the world volume theory is purely bosonic. This is interesting, in particular in view of the the above remarks about the role of supersymmetry in black hole physics. On the other hand, as expected, the absence of a close relation with supersymmetric black holes considerably complicates the analysis of the world 
volume theory in particular. So, for example is is unclear at present how the entropy can be reproduced within world volume theory. Another important issue concerns the stability of the the background. As for all type 0 solutions constructed so far linear stability is guaranteed only as for $A d S$-radii of the order of the string scale. Hence $\alpha^{\prime}$ corrections to the background cannot be excluded on general grounds. However, it has been suggested in (e.g. [7, 8]) that $A d S$ backgrounds are indeed protected due to the vanishing of the Weyl tensor, but further work is clearly needed to decide on the stability. We address some of the issues about the world volume theory which in the infra red is a $1+1$ dimensional CFT. A more in depth analysis, needed for an explicit understanding of the counting of micro states will be referred to future work.

\section{Type 0 Strings}

Type 0 string theory [10] has been discussed in numerous papers. For the analysis presented here [11] and [7] will be most relevant. These papers provide excellent sources for the material used here so that we can limit ourselves to summarise the main points.

\section{Spectrum :}

Here we will be concerned with type $0 B$ string theory which is based on a nonsupersymmetric diagonal GSO projection $\left(\frac{1}{2}\left[1+(-1)^{F+\bar{F}}\right]\right)$, 10

$$
(N S-, N S-) \oplus(N S+, N S+) \oplus(R+, R+) \oplus(R-, R-)
$$

which projects out the fermions in the bulk. Alternatively it can be obtained by projecting type IIB by the discrete symmetry generated by $(-1)^{F_{s}}$, where $F_{s}$ is the space time fermion number. The massless bosonic degrees of freedom in the NS-sector contain a sector identical with the type II theories but include the tachyon in addition. Furthermore the RR fields are doubled. Correspondingly there are two sorts of $D p$ branes for $p$ odd. We denote them by $D p_{ \pm}$. The corresponding boundary states are 111

$$
D p_{ \pm}: \quad|B p, \pm\rangle_{N S N S}+|B p, \pm\rangle_{R R},
$$

where $|B p, \epsilon\rangle_{\eta}$ satisfies

$$
\begin{aligned}
\left(\alpha_{n}^{\mu}-\tilde{\alpha}_{-n}^{\mu}\right)|B p, \epsilon\rangle_{\eta} & =0 \\
\left(\psi_{r}^{\mu}-i \epsilon \tilde{\psi}_{-r}^{\mu}\right)|B p, \epsilon\rangle_{\eta} & =0
\end{aligned}
$$

for $\mu=0, \cdots, p$ and

$$
\begin{aligned}
\left(\alpha_{n}^{\mu}+\tilde{\alpha}_{-n}^{\mu}\right)|B p, \epsilon\rangle_{\eta} & =0 \\
\left(\psi_{r}^{\mu}+i \epsilon \tilde{\psi}_{-r}^{\mu}\right)|B p, \epsilon\rangle_{\eta} & =0
\end{aligned}
$$

for $\mu=p+1, \cdots, 9$ and $\eta \in\{N S N S, R R\}$. Further information about the spectrum of type $O B$ strings comes form the observation [11] that only the combination $D_{p+} D_{p-} \equiv$ $D p_{ \pm}$which is charged only under the untwisted RR-fields has the correct number of degrees of freedom to be interpreted as the S-duals of the fundamental string and the NS-five brane respectively (the $D 3_{ \pm}$is self dual [7]). The twisted fields should then be invariant under S-duality. 
Effective Action:

The string frame low energy effective action up to second order in the tachyon has been worked out in [7] (see also [12])

$$
\begin{aligned}
S= & \frac{1}{2 \kappa_{10}^{2}}\left[\int \sqrt{-G} e^{-2 \phi} R+4\left(e^{-2 \phi} \mathrm{d} \phi, \mathrm{d} \phi\right)-\frac{1}{4}\left(e^{-2 \phi} \mathrm{d} T, \mathrm{~d} T\right)-\frac{m^{2}}{4}\left(e^{-2 \phi} T, T\right)\right. \\
& \left.-\frac{1}{2}\left(e^{-2 \phi} H, H\right)-\frac{1}{2}\left(f_{+}(T) F_{[3]+}, F_{[3]+}\right)-\frac{1}{2}\left(f_{-}(T) F_{[3]-}, F_{[3]-}\right)\right],
\end{aligned}
$$

where $f_{ \pm}(T)=1 \pm T+\frac{1}{2} T^{2}+O\left(T^{2}\right)$ and $(A, B)$ denotes the scalar product of differential forms. All the other fields are set to zero. It was argued in [7] that, provided the effective action is $S$-duality invariant, the exact form should be given by $f_{ \pm}=e^{ \pm T}$.

\section{Non-Dilatonic Solutions}

All non-dilatonic solutions to the type 0 low energy effective action found so far had non-vanishing electric and magnetic RR-flux and correspond to appropriate projections of certain type II BPS solutions. However, as we shall argue below bound states that are purely electric or magnetic also exist but they cannot be obtained by as projections from the type II BPS states.

We consider the six dimensional action obtained from (5) by parametrising $G_{10}$ in terms of the six dimensional Einstein metric

$$
G_{10}=e^{\phi_{6}} g_{6}+e^{2 \nu} d x_{I} d x^{I} .
$$

That is

$$
\begin{aligned}
S_{6}= & \frac{1}{2 \kappa_{6}^{2}}\left[\int \sqrt{-G} R_{6}-\frac{1}{2}\left(\mathrm{~d} \phi_{6}, \mathrm{~d} \phi_{6}\right)-\frac{1}{4}(\mathrm{~d} T, \mathrm{~d} T)-4(\mathrm{~d} \nu, d \nu)-\frac{m^{2}}{4}\left(e^{\phi} T, T\right)\right. \\
& \left.-\frac{1}{2}\left(e^{-2 \phi_{6}} H, H\right)-\frac{1}{2}\left(e^{4 \nu} f_{+}(T) F_{[3]+}, F_{[3]+}\right)-\frac{1}{2}\left(e^{4 \nu} f_{-}(T) F_{[3]-}, F_{[3]-}\right)\right],
\end{aligned}
$$

where $\phi_{6}=\phi-2 \nu, \kappa_{6}^{2}=\kappa_{10}^{2} / L^{4}$ and we have performed the consistent truncation $B_{I J}=F_{I \mu \nu}=H_{I \mu \nu}=0$. The relevant equations of motion that follow from (7) are given by

$$
\begin{aligned}
\nabla^{2} \phi_{6} & =-\frac{m^{2}}{4} T^{2} e^{\phi_{6}}+\left(e^{-2 \phi_{6}} H, H\right) \\
\frac{1}{2} \nabla^{2} T & =-\frac{m^{2}}{2} T e^{\phi_{6}}-\frac{1}{2}\left(e^{4 \nu} f_{+}^{\prime}(T) F_{[3]+}, F_{[3]+}\right)-\frac{1}{2}\left(e^{4 \nu} f_{-}^{\prime}(T) F_{[3]-}, F_{[3]-}\right) \\
8 \nabla^{2} \nu & =-2\left(e^{4 \nu} f_{+}(T) F_{[3]+}, F_{[3]+}\right)-2\left(e^{4 \nu} f_{-}(T) F_{[3]-}, F_{[3]-}\right) \\
0 & =d *\left(H e^{-2 \phi_{6}}\right) \\
0 & =d *\left(F_{[3]+} e^{4 \nu} f_{+}(T)\right) \\
0 & =d *\left(F_{[3]-} e^{4 \nu} f_{-}(T)\right)
\end{aligned}
$$

supplemented with the charge quantisation conditions

$$
Q_{1 \pm}=\frac{1}{4 \pi^{2} g \alpha^{\prime}} \int_{S^{3}} * e^{4 \nu} F_{[3] \pm} \quad \text { and } \quad Q_{5 \pm}=\frac{1}{4 \pi^{2} g \alpha^{\prime}} \int_{S^{3}} F_{[3] \pm},
$$


and similarly for the NS field $H$. For radially symmetric configurations the latter can be implemented by writing the RR-3 form as $\left(r_{i}^{2}=g Q_{i} \alpha^{\prime}\right)$

$$
F_{[3] \pm}=2 r_{5 \pm}^{2} \epsilon_{3}+2 r_{1 \pm}^{2} e^{-4 \nu} * e_{3},
$$

where $e_{3}$ is the volume form of the unit 3-sphere. The 'effective' potential for the tachyon on field and the size of the internal space $\nu$ is then found to bef

$$
\begin{aligned}
V_{e f f}(\nu, T)= & \frac{m^{2}}{2} T^{2} e^{\phi_{6}} \\
& +\frac{4}{V_{3}^{2}}\left(e^{-4 \nu}\left(\frac{r_{1+}^{4}}{f_{+}(T)}+\frac{r_{1-}^{4}}{f_{-}(T)}\right)+e^{4 \nu}\left(r_{5+}^{4} f_{+}(T)+r_{5-}^{4} f_{-}(T)\right)\right),
\end{aligned}
$$

where $V_{3}$ is the volume of the 3 -sphere. In the NS sector one finds a similar potential with $\nu$ replaced by $\phi_{6}$. find

Here we are interested in non-dilatonic solutions. Equating $\frac{\partial}{\partial T} V_{\text {eff }}=\frac{\partial}{\partial \nu} V_{\text {eff }}=0$ we

$$
\begin{aligned}
T & =0 \\
e^{8 \nu} & =\frac{r_{1+}^{4}+r_{1-}^{4}}{r_{5+}^{4}+r_{5-}^{4}} \\
0 & =e^{-4 \nu}\left(-r_{1+}^{4}+r_{1-}^{4}\right)+e^{4 \nu}\left(r_{5+}^{4}-r_{5-}^{4}\right)
\end{aligned}
$$

where we have used the fact that $f_{ \pm}(0)= \pm f_{ \pm}^{\prime}(0)=1$. Note that this is satisfied for any $R R T$ coupling $f_{ \pm}(T)$ that agrees with $1 \pm T$ up to $O(T)$. A particular solution of the the system (12) is given by $r_{i+}=r_{i-}, i=1,5$. This is the solution discussed in a paper by Costa [8]. However, (12) admits yet another set of solutions for $r_{i-}=0\left(r_{i+}=0\right)$. This new class of black hole solutions has electric $\left(D p_{+}\right)$brane charge but no magnetic charge. More precisely one finds

$$
e^{4 \nu}=\frac{r_{1}^{2}}{r_{5}^{2}} \quad \text { and } \quad d s_{6}^{2}=\frac{r^{2}}{r_{1} r_{5}}\left(-\mathrm{d} t^{2}+\mathrm{d} z^{2}\right)+\frac{r_{1} r_{5}}{r^{2}}\left(\mathrm{~d} r^{2}+r^{2} \mathrm{~d} \Omega_{3}^{2}\right) .
$$

Note that we have chosen the solution which is $A d S_{2+1}$ for all $r$ (up to identifications). It corresponds to the near horizon regime of the asymptotically flat solution. The present solution is more suitable for reasons of stability but encodes the essential features of asymptotically flat black holes [14].

The obvious question that arises now is whether the set of all these solutions is connected. This turns out to be the case. Indeed (12) admits a family of solutions with

$$
r_{1+}=r_{5+} \equiv r_{+} \quad \text { and } \quad r_{1-}=r_{5-} \equiv r_{-},
$$

but $r_{+} \neq r_{-}$. This family interpolates between the set of $D p_{ \pm}$black holes discussed by Costa [8] and the the set of electric $\left(D p_{+}\right)$solutions described above. The metric for the interpolating solutions (14) can be obtained from (13) upon substitution

$$
r_{1}^{2}=r_{5}^{2}=\sqrt{r_{+}^{4}+r_{-}^{4}} .
$$

\footnotetext{
${ }^{1}$ To obtain the correct potential one proceeds, as in [13], on the level of the equations of motion. Naive substitution of (10) into the action leads to the wrong sign for the electric potential.
} 


\section{To summarise:}

The low energy effective action for type $0 B$ theory admits a continuous family of solutions interpolating between the $D 1_{ \pm} D 5_{ \pm}$and the $D 1_{+} D 5_{+}$configurations. This deformation preserves the non-dilatonic background.

In the NS sector we parameterise $H$ by

$$
H=2 r_{5}^{2} \epsilon_{3}+2 r_{1}^{2} e^{2 \phi} * \epsilon_{3}
$$

The corresponding solution is as in the type II case

$$
\begin{aligned}
d s_{6}^{2} & =\frac{r^{2}}{r_{1} r_{5}}\left(-\mathrm{d} t^{2}+\mathrm{d} z^{2}\right)+\frac{r_{1} r_{5}}{r^{2}}\left(\mathrm{~d} r^{2}+r^{2} \mathrm{~d} \Omega_{3}^{2}\right) \\
\nu & =0 \\
e^{2 \phi_{6}} & =\frac{r_{5}^{2}}{r_{1}^{2}} .
\end{aligned}
$$

Hence the metric is identical with the RR-solution. One might take this as an indication that that the configuration (17) is the $S$-dual of the $D 1_{+} D 5_{+}$configuration. This is however misleading. As explained in section 2 and [11] the degrees of freedom of the $D 1_{+}$string do not match those of the fundamental string.

To obtain the (extremal) black hole one proceeds as usual [15] by adding momentum in the $z$ direction, that is

$$
\begin{aligned}
d s_{6}^{2} & =\frac{r^{2}}{r_{1} r_{5}}\left(-\mathrm{d} t^{2}+\mathrm{d} z^{2}+K(\mathrm{~d} t+\mathrm{d} z)^{2}\right)+\frac{r_{1} r_{5}}{r^{2}}\left(\mathrm{~d} r^{2}+r^{2} \mathrm{~d} \Omega_{3}^{2}\right) \\
K & =\frac{r_{K}^{2}}{r^{2}}
\end{aligned}
$$

The corresponding five-dimensional black hole is obtained by comparing (18) to the form

$$
d s_{6}^{2}=e^{\frac{2}{3} \lambda} d s_{5}^{2}+e^{2 \lambda}\left(\mathrm{d} z+A_{\mu}^{K}\right)^{2} .
$$

The resulting (near horizon) five dimensional black hole metric is as in the type IIB case

$$
\begin{aligned}
d s_{5}^{2} & =-\frac{1}{f^{\frac{2}{3}}} \mathrm{~d} t^{2}+f^{\frac{1}{3}}\left(\mathrm{~d} r^{2}+r^{2} \mathrm{~d} \Omega_{3}^{2}\right) \\
f & =\frac{r_{1}^{2} r_{5}^{2}}{r^{4}}\left(1+\frac{r_{K}^{2}}{r^{2}}\right) .
\end{aligned}
$$


Stability:

An important point in all type 0 configurations is the stability. For the configuration with unequal electric- and magnetic charge the new issue arising is the mixing tachyonic- and $\nu$-modes (recall $\nu$ describes the internal volume). More precisely the mass matrix becomes

$$
M=8\left(\begin{array}{cc}
\frac{m^{2}}{8}+\frac{1}{r_{1} r_{5}} & \frac{4 q^{2}}{\left(r_{1} r_{5}\right)^{3}} \\
\frac{4 q^{2}}{\left(r_{1} r_{5}\right)^{3}} & \frac{16}{r_{1} r_{5}}
\end{array}\right),
$$

where $q=r_{1+}^{2} r_{5+}^{2}-r_{1-}^{2} r_{5-}^{2}$ is the deformation parameter. On the other hand in the 6 -dimensional Einstein metric the radius of the $A d S_{3}$-factor is given by $l^{2}=r_{1} r_{5}$. Linear stability for the scalar perturbations $T$ and $\nu$ then requires

$$
r_{1} r_{5} \leq-\frac{1}{\lambda_{-}}
$$

where $\lambda_{-}$is the smaller eigenvalue of $M$. For $M$ as in (21) one then obtains

$$
-\frac{1}{4 r_{1} r_{5}} \leq\left(\frac{17}{r_{1} r_{5}}-\frac{1}{4 \alpha^{\prime}}\right)-\sqrt{\left(\frac{15}{r_{1} r_{5}}+\frac{1}{4 \alpha^{\prime}}\right)^{2}+4 \frac{16 q^{2}}{\left(r_{1} r_{5}\right)^{6}}}
$$

where we have used that $m^{2}=-2 / \alpha^{\prime}$. In the case of equal charges this leads top

$$
r_{1} r_{5} \leq\left(4+\frac{1}{2}\right) \alpha^{\prime}
$$

In the opposite limit, that is for $Q_{i-}=0$ one finds

$$
r_{1} r_{5} \leq \frac{1}{2}\left(\frac{137}{129}\right) \alpha^{\prime}
$$

In either case the curvature is of order of $\alpha^{\prime}$ and hence corrections to the metric are to be expected unless the backgrounds is protected. It was observed before (e.g. [7, 8]) that this applies to $A d S_{2+1} \times S^{3}$ backgrounds provided these corrections can be written in terms of the Weyl tensor only.

For the F1NS5 configuration there is no mixing between the tachyon and $\nu$ and consequently linear stability requires

$$
\frac{\alpha^{\prime}}{2} \geq e^{\phi_{6}} r_{1} r_{5}=g Q_{5} \alpha^{\prime}
$$

where $g$ is the string coupling.

\section{World volume theory}

The metric of the $D 1_{+} D 5_{+}$black hole described in the last section is identical with type IIB. The entropy and the Hawking decay rate are therefore also identical. If the

\footnotetext{
${ }^{2}$ See also 9 for an extensive discussion of stability for equal charges.

${ }^{3}$ The extra $\frac{1}{2}$ compared to [8] comes when one takes into account that in $A d S_{2+1}$ stability is compatible with $m^{2}=-1 / l^{2}$.
} 
black hole background described in the last section is a consistent ground state of string theory then it should be possible to reproduce these features form the world volume theory of the $D 1_{+} D 5_{+}$system. This task is, however, complicated by the absence of supersymmetry or equivalently, by the presence of the tachyon which prevents us from interpolating the background to curvature much smaller than $1 / \alpha^{\prime}$. As a result the predictions for the world volume theory can be trusted only up to $\alpha^{\prime}$ - corrections.

As a first step towards a detailed understanding of the world volume theory we

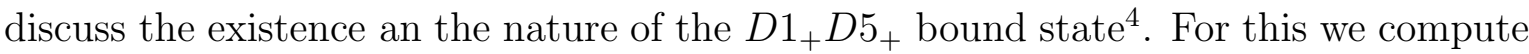
the open string loop amplitude in terms of the corresponding boundary states [11]. For the NS-NS sector we have

$$
\left\langle B p, \epsilon\left|e^{-l H_{c}^{o s c}}\right| B s, \epsilon^{\prime}\right\rangle_{N S N S}=\operatorname{Tr}_{N S}\left(e^{-2 l L_{0}}\left(\epsilon \epsilon^{\prime}\right)^{F} \prod_{\mu=p+3}^{s+2}(-1)^{B_{\mu}}\right),
$$

where

$$
(-1)^{B_{\mu}} \alpha_{m}^{\nu}(-1)^{B_{\mu}}= \begin{cases}\alpha_{m}^{\nu} & \mu \neq \nu \\ -\alpha_{m}^{\nu} & \mu=\nu\end{cases}
$$

and the same relation for the fermionic modes $\psi_{m}^{\nu}$. Here $H_{c}^{o s c}$ is the oscillator part of the closed string Hamiltonian. The RR sector works analogously. The relevant amplitudes are then given by

$$
\begin{aligned}
& \left\langle B p,+\left|e^{-t H_{c}^{o s c}}\right| B p,+\right\rangle=\frac{f_{3}^{8}(\tilde{q})}{f_{1}^{8}(\tilde{q})}-\frac{f_{2}^{8}(\tilde{q})}{f_{1}^{8}(\tilde{q})} \quad \text { and } \\
& \left\langle B p,+\left|e^{-t H_{c}^{o s c}}\right| B s,+\right\rangle=\frac{f_{3}^{8-\nu}(\tilde{q})}{f_{1}^{8-\nu}(\tilde{q})} \frac{f_{4}^{\nu}(\tilde{q})}{f_{2}^{\nu}(\tilde{q})} ; p<s,
\end{aligned}
$$

where $\nu=s-p, \tilde{q}=e^{-2 \pi l}$ and the functions $f_{i}(\tilde{q})$ can be found in [16]. In order to obtain the potential between two branes separated by a distance $r$, we perform the remaining integral over the momenta leading to

$$
\begin{aligned}
& V_{p, p}(r)=-\int \frac{\mathrm{d} t}{2 t}\left(8 \pi^{2} \alpha^{\prime} t\right)^{-\frac{p+1}{2}} e^{-t \tilde{r}^{2}}\left(\frac{f_{3}^{8}(q)}{f_{1}^{8}(q)}-\frac{f_{4}^{8}(q)}{f_{1}^{8}(q)}\right) \text { and } \\
& V_{p, s}(r)=-\int \frac{\mathrm{d} t}{2 t}\left(8 \pi^{2} \alpha^{\prime} t\right)^{-\frac{p+1}{2}} e^{-t \tilde{r}^{2}}\left(\frac{f_{3}^{8-\nu}(q)}{f_{1}^{8-\nu}(q)} \frac{f_{2}^{\nu}(q)}{f_{4}^{\nu}(q)}\right)
\end{aligned}
$$

where $\tilde{r}^{2}=r^{2} /\left(2 \pi \alpha^{\prime}\right), q=e^{-\pi t}$ and we have expressed the result in terms of the more familiar open string one loop amplitude via the substitution $l=1 /(2 t)$. At short distances which corresponds to $t \rightarrow \infty$, the potential between $D 1_{+}$and $D 5_{+}$branes is then obtained by expanding the integrand i.e.

$$
\frac{f_{3}^{8}(q)}{f_{1}^{8}(q)}-\frac{f_{4}^{8}(q)}{f_{1}^{8}(q)}=16+O(q) \quad \text { and } \quad \frac{f_{3}^{8-\nu}(q)}{f_{1}^{8-\nu}(q)} \frac{f_{2}^{\nu}(q)}{f_{4}^{\nu}(q)}=2^{\nu / 2}+O(q) \text {. }
$$

As they stand the $t$-integrals are, however, not well defined due to the divergence at $t \rightarrow 0$. Of course, the full string theory result should be finite. As explained in [17] the divergences in (31) are related to the presence of a tachyonic mode in the closed string

\footnotetext{
${ }^{4}$ We refer the reader to [8] for the analogous analysis of the $D p_{ \pm}$bound states.
} 
sector and the coefficients of the counter terms needed to cancel the small $t$ divergences will depend on the details of the tachyon condensation. Fortunately for $V_{1,1}$ and $V_{1,5}$ it turns out that the counter term is $r$ independent and can therefore be discarded. For $r^{2}<<\alpha^{\prime}$ and $\Lambda=\sqrt{\alpha^{\prime}}$ the resulting potentials read

$$
\begin{aligned}
V_{1,1}(r) & =-\frac{\tilde{r}^{2}}{\pi^{2} \alpha^{\prime}} \log \left(\tilde{r}^{2}\right) \text { and } \\
V_{1,5}(r) & =\frac{1}{4} V_{1,1}(r) .
\end{aligned}
$$

In contrast to the $D 3_{+}$system [0], the potential for $D 1_{+} D 1_{+}$and for $D 1_{+} D 5_{+}$is attractive for small distances. This is consistent with the existence of a conformal (non-dilatonic) gravitational background. For a $D 5_{+} D 5_{+}$-pair the potential takes the form

$$
V_{5,5}(r)=c_{0}+c_{1} \tilde{r}^{2}+c_{2} \tilde{r}^{4}+\frac{\tilde{r}^{6}}{3 ! 64 \pi^{6}\left(\alpha^{\prime}\right)^{3}} \log \left(\tilde{r}^{2}\right) .
$$

As mentioned above the constants $c_{i}$ depend on the details of the tachyon condensation which is not understood in detail so far. We are therefore unable to fix them at present. The configuration with $\tilde{r}=0$ (i.e. all $D 5_{+}$on top of each other) is an extremum of $V_{5,5}(r)$ for all values of the $c_{i}$. However, the sign of $c_{1}$ determine whether this configuration is stable. If the stable configuration is at $r>0$ then, unless $Q_{5}<<Q_{1}$ most of the $(1,1)$ and the $(1,5)$ strings will be massive and will not contribute significantly to the entropy. If, on the other hand all of the $D 5_{+}$branes are on top of each other then the $(1,1)$ and the $(1,5)$ strings will be massless. However, a naive counting of the massless degrees of freedom still only accounts for $2 / 3$ of the Beckenstein-Hawking entropy. Indeed the $4 Q_{1} Q_{5}$ bosonic degrees of freedom lead to a central charge [15]

$$
c=4 Q_{1} Q_{5}
$$

which is to be compared with $c=6 Q_{1} Q_{5}$ in the type IIB case. The difference is, of course, due to the absence of fermions in the world volume theory. However, because the world volume theory is interacting even in the infrared the arguments presented above are probably too naive and a more quantitative analysis of the world volume theory will be needed.

\section{Discussion}

In this paper we have described a new class of non-dilatonic type 0 backgrounds distinguished by the fact that only one of the two RR-fluxes $\left(F_{[n] \pm}\right)$ is active. Unlike previous non-dilatonic backgrounds the solutions described here cannot be obtained by a projection of some type II BPS state. In particular we constructed an $A d S_{2+1}$ (BTZ) black hole from 'electric' $D 1_{+} D 5_{+}$branes. A novel feature of this configuration is the absence of fermions in the world volume theory. The existence of a non-dilatonic gravity dual suggests, however, that in the IR the world volume theory should nevertheless flow to a conformal field theory. In this paper we presented but a qualitative analysis the world volume theory. From a more detailed analysis of the interactions we may hope to gain valuable insight into the role of fermions in the microscopic theory of black holes. For 
that purpose the observation that the system can be continuously deformed form the $D 1_{ \pm} D 5_{ \pm}$to the $D 1_{+} D 5_{+}$configuration may prove useful. As all type 0 configurations a tachyonic instability develops when the curvature decreases below the string scale. It seems reasonable to believe that this instability should have an analogue in the world volume theory, for example, in form of a large $N$ phase transition. A similar proposal has been made recently in 18 for the world volume theory of the non-supersymmetric $D 3$-brane system of type 0 theory. It would be interesting to analyse the $D 1 D 5$-brane system from this point of view.

\section{Acknowledgements:}

The author would like to thank the Department of Mathematics at Kings College London for hospitality during the final stages of this work. This work was supported by a Swiss Government TMR Grant, BBW Nr. 970557.

\section{References}

[1] A. Strominger and C. Vafa, Phys. Lett. B379 (1996) 99, hep-th/9601029

A. Sen, Mod. Phys. Lett. A 10 (1995) 2081, hep-th/9504147

M. Cvetič and A.A. Tseytlin, Phys. Rev. D53 (1996) 5619, Erratum-ibid. D55 (1997) 3907, hep-th/9512031

G. Horowitz and A, Strominger, Phys. Rev. Lett. 77 (1996) 591, hep-th/9602051.

[2] D. Brown and M. Henneaux, Commun. Math. Phys. 104 (1986) 207.

[3] A. Strominger, JHEP 9802 (1998) 009, hep-th/9712251

D. Birmingham, I. Sachs and S. Sen, Phys.Lett. B424 (1998) 275, hep-th/9801019

M. Banados, T. Brotz, M. Ortiz, Nucl. Phys. B545 (1999) 340.

[4] O. Coussaert, M. Henneaux, P. van Driel, Class. Quant. Grav. 12 (1995) 2961, gr-qc/9506019

R. Emparan and I. Sachs, Phys. Rev. Lett. 81 (1998) 2408, hep-th/9806122.

[5] S. Carlip, Class. Quant. Grav. 15 (1998) 3609; hep-th/9806026

[6] N. Seiberg and E. Witten, JHEP 9904 (1999) 017, hep-th/9903224.

[7] I. R. Klebanov and A. A. Tseytlin, Nucl. Phys. B546 (1999) 155, hep-th/9811035;

Nucl. Phys. B547 (1999) 143, hep-th/9812089; JHEP 9903 (1999) 015, hepth/9901101.

[8] M. S. Costa, hep-th/9903128.

[9] Y. S. Myung, H. W. Lee and Jin Young Kim, hep-th/9907024.

[10] L. Dixon and J. Harvey, Nucl. Phys. B274 (1986) 93

N. Seiberg and E. Witten, Nucl. Phys. B276 (1986) 272. 
[11] O. Bergman and M. Gaberdiel, Nucl. Phys. B499 (1997) 183, hep-th/9701137; hep-th/9906055.

[12] M. R. Garousi, hep-th/9901085.

[13] M. Cvetič and A.A. Tseytlin, Nucl. Phys. B416 (1994) 137, hep-th/9307123.

[14] K. Sfetsos and K. Skenderis, Nucl.Phys. B517 (1998) 179, hep-th/9711138 I. Sachs, Proceedings, PASCOS98, hep-th/9804173.

[15] C. G. Callan and J. M. Maldacena, Nucl.Phys. B472 (1996) 591, hep-th/9602043.

[16] J. Polchinski and Y. Cai, Nucl.Phys. B296 (1988) 91.

[17] A. A. Tseytlin and Z. Zarembo, hep-th/9902095.

[18] I. R. Klebanov, hep-th/9906220. 